\title{
HAFTUNG
}

\section{Für den Fall des Falles}

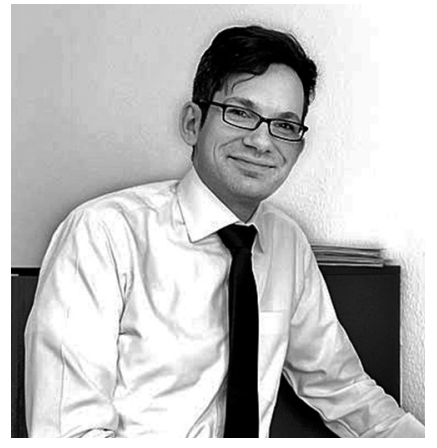

VON BENJAMIN LIEDY

Dr. Benjamin Liedy ist Rechtsanwalt in der Kanzlei Reith Schick \& Partner, Stuttgart. Zu seinen Schwerpunkten gehört die Beratung von Non-Profit-Organisationen in vereins-, stiftungs- und gemeinnützigkeitsrechtlichen Fragen.

benjamin.liedy@reithschick.de

\author{
Die Frage, wie handelnde Personen in Vereinen \\ haften, wenn durch Managementfehler \\ "etwas schief geht", sorgt gerade bei kleineren \\ Vereinen im gemeinnützigen Bereich öfter für \\ Verunsicherung. Nicht zuletzt auch deshalb \\ wurde die Innenhaftung von Vereinsmitgliedern \\ im Jahre 2009 vom Gesetzgeber geregelt.
}

Im folgenden Beitrag werden die verschiedenen Haftungsbereiche dargestellt und die Möglichkeiten aufgezeigt, diese abzusichern. Grundsätzlich unterscheidet man beim Haftungsrecht im Zusammenhang mit dem Verein zwei Bereiche: Zum einen ist dies die Haftung des Vereins für seine Organe und Hilfspersonen; zum anderen stellt sich die Frage der (persönlichen) Haftung der handelnden Personen.

\section{Haftung des Vereins oder der Körperschaft}

Der Verein als juristische Person haftet gemäß $\ 31$ BGB grundsätzlich für die Tätigkeit seiner Organe - in Ausführung ihres Vereinsamtes.

Dabei wird der Organbegriff von der Rechtsprechung recht weit ausgelegt. (1) Das heißt nicht nur Handlungen des Vorstandes, sondern auch diejenigen von Funktionsträgern, die nicht explizit in der Satzung erwähnt werden, sind von $\$ 31$ BGB umfasst. Diese Haftung kann auch nicht durch Satzung begrenzt werden. (2)

Darüber hinaus gelten auch für Vereine die $\$ \mathbb{S} 278,831$ BGB, die - grob gefasst - regeln, dass sich der Verein schuldhaftes Handeln von Personen, die er zur Erfüllung oder Verrichtung seiner Aufgaben beauftragt, zurechnen lassen muss.

\section{Persönliche Haftung der handelnden Personen}

Auch bei der Tätigkeit für einen Verein bleibt eine persönliche Haftung der (Organ-) Mitglieder bestehen. Hier sind ebenfalls zwei Bereiche zu unterscheiden: Zum einen geht es um die Haftung nach außen, also gegenüber Außenstehenden. Zum anderen ist von Bedeutung, wie die Organmitglieder für ihre Tätigkeit gegenüber dem Verein haften.

\section{Haftung gegenüber Dritten}

Die handelnden Organmitglieder - also der Vorstand - haften Außenstehenden gegenüber grundsätzlich nach den allgemeinen Rechtsvorschriften persönlich. In den verschiedenen Rechtsformen gibt es aber Unterschiede je nach dem, aus welchem Grund die handelnde Person in Anspruch genommen wird. Hier liegt der wesentliche Unterschied zwischen dem nicht rechtsfähigen und dem eingetragenen Verein. Im nicht rechtsfähigen Verein haften die handelnden Mitglieder für alle ihre Handlungen. Das bedeutet insbesondere, dass sie beim Abschluss eines Vertrages dem Vertragspartner neben dem Verein persönlich haften. Für sämtliche vertraglichen Ansprüche sind diejenigen, die gegenüber dem Vertragspartner gehandelt haben, also auch verantwortlich. Sei es für den Anspruch auf die Gegenleistung oder bei Schaden- 
ersatzansprüchen (etwa wenn Veranstaltungen kurzfristig nicht durchgeführt werden können). Zudem haften die Mitglieder für Schäden, die durch sogenannte »unerlaubte Handlungen « (dazu sogleich) entstehen. Demgegenüber haftet beim eingetragenen Verein für die vertraglichen Verbindlichkeiten nur der Verein an sich. (3) Die handelnden (also vertragsschließenden) Mitglieder können hierfür nicht in die Verantwortung genommen werden. Allerdings haben die Mitglieder hier wie auch im nicht rechtsfähigen Verein für sogenannte »unerlaubte Handlungen « einzustehen. Beispiele hierfür sind:

- Sie verursachen in Ausübung einer Vorstandstätigkeit einen Verkehrsunfall.

- Sie verletzen mit einer Publikation ein Urheberrecht.

- Bei einer Veranstaltung kommen Teilnehmer zu Schaden, weil die Räume nicht verkehrssicher waren und der Vorstand dies geduldet hat.

- In einer Pflegeeinrichtung werden im Wissen des Vorstands des Trägervereins systematisch Hygienegrundsätze missachtet.

\section{«Kann der Verein die}

\section{Sozialversicherungsbeiträge}

nicht zahlen, muss der Vorstand

notfalls die Nettolöhne kürzen"

Das sind nur einige Beispiele. Grundsätzlich muss man feststellen, dass die Rechtsprechung sehr schnell sogenannte Organisationspflichtverletzungen annimmt: Immer wenn Mängel in der Vereinsorganisation den Schadenseintritt durch Handlungen anderer Mitglieder oder sonstiger vom Verein beauftragter Dritter begünstigt haben, besteht ein Haftungsrisiko für den Vorstand. Die Haftung des Vereins nach \ 31 BGB nimmt dabei dem Vorstand diese Haftung nicht ab. Vielmehr wird dem Geschädigten dadurch ein zweiter Schuldner verschafft: der Verein. Verein und Vorstandsmitglied haften also beide.

Wenn man sich beim mehrgliedrigen Vorstand über eine Maßnahme nicht einig ist, muss das Mitglied, das ein herangezogen werden. (4) sigkeit indiziert. (5) haftungsträchtiges Problem sieht, alles rechtlich mögliche tun, um den Beschluss abzuändern. Ansonsten bleibt es auch bei seiner persönlichen Haftung.

Von diesen Grundsätzen der Außenhaftung lässt sich nicht durch Satzungsbestimmungen abweichen.

\section{Steuerliche und sozialrechtliche Haftung}

Neben den zivilrechtlichen Haftungsrisiken für Vereinsvorstände werden oft zwei andere Bereiche nicht beachtet: die Haftung für die Erfüllung steuerlicher und sozialversicherungsrechtlicher Pflichten gegenüber den Arbeitnehmern.

Gemäß $\mathbb{3} 34$ Abs. 1 AO haften bei Körperschaften deren gesetzliche Vertreter für die Erfüllung der steuerlichen Pflichten. Damit ist grundsätzlich der nach $\int 26$ BGB vertretungsberechtigte Vorstand in der Verantwortung.

Für den nicht eingetragenen Verein kommt $\mathbb{S} 34$ Abs. 2 AO zum Tragen, wonach die Mitglieder für die Erfüllung der Pflichten verantwortlich sind, solange kein Vorstand besteht. Auch »einfache" Mitglieder, denen steuerlich relevante Geschäfte zur Führung - wie

Wege einer Aufgabenteilung delegiert werden. Voraussetzung dafür ist nach dem Bundesfinanzhof aber, dass diese Delegation ausdrücklich und schriftlich erfolgt sowie, dass der Gesamtvorstand jederzeit darüber im Bilde ist, dass die Pflichterfüllung auch funktioniert. Bei ersten Zweifeln, dass dem nicht mehr so ist, muss der restliche Vorstand wiederum für die ordnungsgemäße Pflichterfüllung sorgen. (6)

In dem Bereich der Sozialversicherungsbeiträge besteht eine ähnliche Haftungskonstellation. Hier ergibt sich aus dem $\mathbb{S} 823$ Abs. 2 BGB i. V. m. $\mathbb{S}$ $266 a, \mathbb{S} 14 \mathrm{StGB}$, dass die vertretungsberechtigten Vorstände für die Abführung der Arbeitnehmerbeiträge haften. Dabei ist zu beachten, dass die Beiträge bereits mit Aufnahme der Beschäftigung fällig werden. Es wird nicht etwa an die Zahlung des Lohnes angeknüpft. Auch wenn kein Entgelt geflossen ist, besteht also diese Verpflichtung. Wird absehbar, dass eine Bedienung der Beiträge nicht möglich ist, muss der Vorstand geeignete Maßnahmen ergreifen, um die Zahlungsfähigkeit herzustellen - etwa auch durch Kürzung der Nettolöhne. (7) Wichtig ist zur Vermeidung der Haftung, nicht kommentarlos an die Sozialversicherung zu zahlen, sollten die Mittel nicht für die Gesamtbeiträge ausreichen. Da die Haftung nur für Arbeitnehmeranteile greift, sollte ausdrücklich auf diese Anteile gezahlt werden. (8)

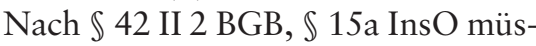
sen die Vorstände bei Überschuldung oder Zahlungsunfähigkeit des Vereins unverzüglich die Insolvenz anmelden. Wird dies versäumt, haften sie den Gläubigern für den daraus entstehenden Schaden.

die Kassenführung - zugewiesen wurde und die nach außen auftreten, können

In Verbindung mit $\mathbb{S} 69$ AO kann dies zu erheblichen Haftungsrisiken für den betroffenen Vorstand führen. Nach dieser Norm haftet dieser für die nicht eingetriebenen Steuern und Zinsverluste, sofern die Verletzung der Pflichten grob fahrlässig oder vorsätzlich war. Dabei geht der Bundesfinanzhof auch für kleine Vereine davon aus, dass die Verletzung steuerlicher Pflichten eine grobe Fahrläs-

Die Rechtsprechung erwartet also vom Vorstand eines Vereins, dass er sich seiner steuerlichen Pflichten bewusst ist. Diese Pflichten können zum Teil auf andere Vorstandsmitglieder im

\section{Haftung gegenüber dem Verein}

Neben dieser Außenhaftung können die Organe gegenüber dem Verein selbst haften müssen oder aber der Verein muss sie eventuell von der Außenhaftung freistellen.

Zentrale Regelung hierfür ist der 2009 geschaffene $\mathbb{S} 31$ a BGB. Diese Norm wurde ins Gesetz eingeführt, weil man Personen, die sich ehrenamtlich (bis zu 500 Euro im Jahr) engagieren, keine übermäßigen Haftungsrisiken aufbürden wollte. (9) \$31a beschränkt die Haftung des geschäftsführenden Vorstands dem Verein gegenüber auf Fehler, die mit Vor- 
satz oder grober Fahrlässigkeit bei der Geschäftsführung erfolgt sind. Die Außenhaftung betrifft $\$ 31$ a dagegen nicht.

Darüber hinaus gibt Absatz 2 dieser Vorschrift dem Vereinsvorstand einen sogenannten Freistellungsanspruch gegenüber dem Verein für den Fall, dass er von Dritten in Haftung genommen wird.

Konkret bedeutet dies: Der Vorstand kann vom Verein verlangen, diese Forderung eines Dritten zu erfüllen oder dem Vorstandsmitglied zu erstatten. Wirtschaftlich wird damit dem ehrenamtlichen Vorstand die beschriebene Außenhaftung solange abgenommen, wie der Verein liquide ist. Wird der Verein aber insolvent, haftet der Vorstand weiterhin.

\section{Versicherung}

Um die dargestellten Haftungsrisiken für die Vorstände und Mitglieder wirtschaftlich zu minimieren, ist ein Versicherungsschutz unverzichtbar. Dabei gibt es verschiedene Formen, die von Verein und Mitglied abgeschlossen werden können. Die vier wichtigsten Policen seien erwähnt:

- Privathaftpflicht: Nach Auffassung der Versicherer tritt die Privathaftpflichtversicherung nicht für Organe im engen Sinn und ehrenamtliche Tätigkeit nach öffentlich-rechtlichen Vorschriften ein. Nur für »Tätigkeiten, die nicht mit einer besonderen Verantwortung verbunden sind «, bestehe Versicherungsschutz. (10) Die Absicherung für Vereinsvorstände durch diese Versicherung ist also lückenhaft.

- Vereinshaftpflicht, Vermögensschadenhaftpflicht: Eine Vereinshaftpflichtversicherung deckt die beschriebenen Risiken für den Verein ab. Die handelnden Personen (Vorstände) sind in einer solchen Police in der Regel mit umfasst. Es werden grundsätzlich Sach-und Personenschäden gedeckt, reine Vermögensschäden müssen meist extra abgesichert werden. Diese Versicherung ist für Vereine mit relevantem Tätigkeitsumfang nach außen unbedingt zu empfehlen.

- Veranstalterhaftpflicht: In der Vereinshaftpflichtversicherung sind satzungsmäßige Veranstaltungen in vereinsüblichen Umfang mit umfasst. Für besondere Veranstaltungen, die den sonst bei dem Verein üblichen Rah- men übersteigen, ist die gesonderte Vereinbarung einer Veranstalterhaftpflichtpolice zu empfehlen.

- D\&O Versicherung: Die D\&O (»director's and officer's liability «) Versicherung ist eine speziell auf Organe von Körperschaften ausgerichtete Vermögensschadenhaftpflichtversicherung. Sie deckt neben der Außenhaftung des Organs auch die Innenhaftung gegenüber dem Verein ab (11), was die Vereinspolice regelmäßig nicht leistet. Und ein ganz wesentlicher Vorteil ist, dass es hier Policen am Markt gibt, die auch die Haftung außerhalb des Zivilrechts abdecken $(\mathbb{S} 69$ AO, $\mathbb{S}$ 42 BGB). Versicherungsnehmer werden normalerweise die Körperschaften für ihre Organe. Am Markt werden auch speziell für kleinere und mittlere Vereine solche Policen angeboten. Vorsicht ist allerdings wegen des Problems der Nachhaftung geboten. Die D\&O Versicherungen knüpfen den Versicherungsfall meistens an den Zeitpunkt der Geltendmachung von Schadenersatzansprüchen gegenüber dem Versicherungsnehmer. Besteht zu diesem Zeitpunkt die Police nicht mehr, sind die Ansprüche aber noch nicht verjährt, besteht eine erhebliche Deckungslücke. (12)

\section{Anmerkungen}

(1) BGH NJW 1968, 391, 392.

(2) Sauter/Schweyer/Waldner, Der eingetragene Verein, 19. Auflage 2010, Rdnr. 292d.

(3) Die hier dargestellten Grundsätze gelten sinngemäß auch für den Vorstand einer rechtsfähigen Stiftung.

(4) Vgl. Rüsken in Klein AO 10. Aufl. 2009 \34 Rn. 8 f.

(5) BFH NJW RR 2003, 1117, 1119.

(6) Vgl. ausführlich Reichert Vereinsrecht 12. Auflage 2010 Rdnr. 3763 ff.

(7) Vgl. Reichert Rdnr. 3749 ff.

(8) Vgl. Ehlers NJW 2011, 2690, 2693.

(9) Der Ersatz nachgewiesener Auslagen hindert dabei die Ehrenamtlichkeit nicht. BT-Drs. 16/13537 S. 6.

(10) Internet http://www.klipp-und-klar. de/dateien/dokumente/versicherungen/VKK_Ehrenamt_April_2012.pdf [16.10.2012].

(11) Vgl. Muschner in NK VVG 2. Aufl. 2011 \$ 43 Rn. 21

(12) Vgl. Schimikowski in NK VVG 2. Aufl. 2011 \ 100 Rn. 14.

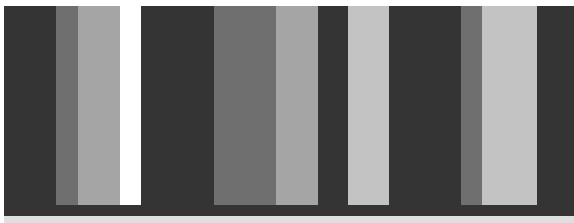

\section{Das Standardwerk seit über 30 Jahren jetzt vollständig überarbeitet und aktualisiert!}

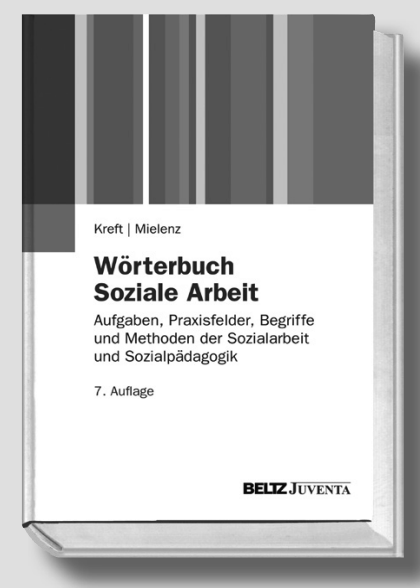

Dieses ausbildungs- und praxisbezogene Standardwerk gibt zuverlässig Auskunft über Ziele, Aufgaben, Arbeitsfelder und Methoden der Sozialen Arbeit - von A bis Z.

Für die 7. Auflage ist das Wörterbuch wieder vollständig überarbeitet und aktualisiert worden. Es enthält jetzt 310 Stichwörter sowie ausführliche Verzeichnisse der Organisationen/ Institutionen und Fachzeitschriften der Sozialen Arbeit.

Das Wörterbuch ist ein Nachschlagewerk und zugleich eine Einführung in die Soziale Arbeit; es empfiehlt sich für Ausbildung und Praxis, will aber auch diejenigen ansprechen, die nicht unmittelbar in der Sozialen Arbeit tätig sind, aber immer wieder mit ihr zu tun haben (Lehrer, Polizisten, Ausbilder in der beruflichen Bildung, Arbeitsvermittler, Stadtplaner, Mediziner, Psychologen, Therapeuten Richter und Fachanwälte) - denn Sozialpolitik bestimmt das ganze Leben.

7., vollständig überarbeitete und aktualisierte Auflage 2013

1088 Seiten, Hardcover

$€ 68$,-

ISBN 978-3-7799-2082-3

\section{www.juventa.de}

\section{BELTZJUVENTA}

